\title{
Symptomatic life-threating hyponatremia complicating severe COPD exacerbation, pulmonary edema and pulmonary hypertension-a critical role for conivaptan
}

\author{
Macaulay Amechi Chukwukadibia Onuigbo ${ }^{1,2^{*}}$, Muhammad A Rishi ${ }^{3}$, Nneoma Agbasi ${ }^{4}$, Mark Henry \\ Alon $^{5}$, Yasser Sammour 6 , Thuyanh Culver ${ }^{5}$, Adel Zurob ${ }^{3}$, Abdul Khan' ${ }^{2}$, Emeka Amadi ${ }^{5}$ \\ ${ }^{1}$ Mayo Clinic College of Medicine, Rochester, MN, USA \\ ${ }^{2}$ Department of Nephrology, Mayo Clinic Health System, Eau Claire, WI, USA \\ ${ }^{3}$ Department of Pulmonary and Critical Care Medicine, Mayo Clinic Health System, Eau Claire, WI, USA \\ ${ }^{4}$ Practice Improvement Team, NELFT NHS Foundation Trust, Basildon, Essex, UK \\ ${ }^{5}$ Department of Hospital Medicine, Mayo Clinic Health System, Eau Claire, WI, USA \\ ${ }^{6}$ Faculty of Medicine, Ain Shams University, Cairo, Egypt
}

\section{A R T I C L E I N F 0}

\section{Article Type:}

Case Report

\section{Article History:}

Received: 14 November 2017

Accepted: 23 January 2018

Published online: 14 February 2018

\section{Keywords:}

Acutely decompensating heart

failure

COPD

Conivaptan

Heart failure

Hyponatremia

Vasopressin antagonists

\begin{abstract}
A B S T R A C T
Hyponatremia, the most common electrolyte abnormality in hospitalized patients, is associated with increased morbidity and mortality. Phase 3 clinical trials and subsequent studies including the Study of Ascending Levels of Tolvaptan in Hyponatremia 1 and 2 (SALT 1 and 2) clearly demonstrated the efficacy of vasopressin antagonists in increasing plasma sodium levels. The "vaptans", oral tolvaptan and intravenous conivaptan, are vasopressin antagonists but as recently as 2015, there remained conflicting recommendations for their use by different expert committees in patients with hyponatremia. This circumstance was blamed on limited patient experiences and limited research data. We recently encountered worsening life-threatening symptomatic hyponatremia, unresponsive to hypertonic $3 \%$ saline infusion, and impending respiratory failure in a 62-year old obese Caucasian male patient who was further complicated by advanced chronic obstructive pulmonary disease (COPD), pulmonary hypertension and acutely decompensating diastolic heart failure, albeit with stable CKD II creatinine levels. Intravenous loop diuretics may have helped with heart failure but potentially would have aggravated the already critically low sodium levels. He demonstrated a brisk response to intravenous conivaptan administration. Intravenous conivaptan is sine qua non the absolute ideal therapeutic agent for acutely decompensating congestive heart failure with concurrent life-threatening hyponatremia.
\end{abstract}

Implication for health policy/practice/research/medical education:

We report a 62-year-old obese hypertensive Caucasian male patient with advanced COPD, pulmonary hypertension and acutely decompensating diastolic heart failure who presented with severe symptomatic life-threatening hyponatremia. Hypertonic 3\% saline infusion did not improve hyponatremia even at $40 \mathrm{cc} / \mathrm{h}$ infusion rate and the patient was quickly experiencing worsening pulmonary edema with respiratory distress and imminent need for ventilatory support. The swift use of intravenous conivaptan, a vasopressin antagonist, with close 4-hourly monitoring of the serum sodium levels resulted in a prompt aquaresis with resolution of symptoms of pulmonary edema, fluid retention and simultaneous correction of life-threatening symptomatic hyponatremia. Vasopressin antagonists can be a life-saving therapeutic option in such critical clinical circumstances.

Please cite this paper as: Onuigbo MAC, Rishi MA, Agbasi N, Alon MH, Sammour Y, Culver T, et al. Symptomatic lifethreating hyponatremia complicating severe COPD exacerbation, pulmonary edema and pulmonary hypertension-a critical role for conivaptan. J Renal Inj Prev. 2018;7(2):98-102. DOI: 10.15171/jrip.2018.23. 


\section{Introduction}

Hyponatremia is the most common electrolyte abnormality in hospitalized patients and is associated with increased morbidity and mortality (1-6). Furthermore, experience from phase three trials and subsequent studies including the Study of Ascending Levels of Tolvaptan in Hyponatremia 1 and 2 (SALT 1 and 2), clearly demonstrated that the efficacy of vasopressin antagonists in increasing the plasma sodium level is unquestionable (1-4).

The vaptans, oral tolvaptan and intravenous conivaptan, are vasopressin antagonists (7). As recently as 2015, there existed conflicting recommendations for the use of vaptans by different expert committees in patients with hyponatremia and this was blamed on limited patient experiences and limited research data (7). Nevertheless, there is now accumulating evidence-base in the literature to strongly support the use of the vaptans in the management of severe symptomatic treatment-resistant hyponatremia especially in association with heart failure, advanced chronic obstructive pulmonary disease (COPD) and/or pulmonary hypertension (6,8-11).

Vasopressin antagonists block the vasopressin receptors $\mathrm{V} 1$ and V2. The V2 receptor is located primarily in the basolateral membrane of collecting duct cells and V2 blockage leads to aquaresis. V2 receptor inhibition culminates in the prevention of the insertion of water channels into the apical membrane, which thereby inhibits the reabsorption of water and the generation of concentrated urine thereby producing prompt aquaresis and polyuria (7). Conivaptan is twice as potent as tolvaptan as an inhibitor of the V1 receptor, but tolvaptan is 2.5 times as potent an inhibitor of the $\mathrm{V} 2$ receptor. Thus, conivaptan is a nonselective vasopressin inhibitor, whereas tolvaptan is a more selective V2 inhibitor. Tolvaptan and conivaptan (with the latter blocking both the V1 and V2 receptors) have garnered approval for the treatment of euvolemic and hypervolemic hyponatremia in the United States (7).

We recently managed a 62-year-old obese hypertensive Caucasian male patient with known advanced COPD, pulmonary hypertension and diastolic heart failure who presented with severe symptomatic and life-threatening hyponatremia. Hypertonic 3\% saline infusion did not improve hyponatremia even at $40 \mathrm{cc} / \mathrm{h}$ infusion rate and the patient was quickly experiencing worsening pulmonary edema with respiratory distress and imminent need for ventilatory support. The use of intravenous conivaptan loomed as a most appropriate salvage therapeutic option. Here is our experience.

\section{Case Presentation}

In 2017, our nephrology service was consulted for severe hyponatremia. The patient was an obese hypertensive 62year old Caucasian male smoker with a history of recurrent COPD exacerbations and diastolic congestive heart failure. Past medical history was significant for multiple admissions with COPD exacerbation and congestive diastolic heart failure, chronic bilateral lower extremity edema, hyponatremia exacerbations in the past, previous lumbar fusion L4-L5, hypertension, ongoing tobacco use after quitting in 2014, glucose intolerance and proximal left femoral-popliteal bypass.

$\mathrm{He}$ was admitted following the sudden loss of consciousness or syncope and a fall with some facial injury that required ENT surgical intervention in the emergency department. His serum sodium had plummeted from 134 $\mathrm{mmol} / \mathrm{L}$ four days earlier to $116 \mathrm{mmol} / \mathrm{L}$ on admission. He had just returned from the store when he fell almost without warning and was transferred to the emergency department. He had admitted to drinking "a couple of beers daily". However, according to his sister, he drinks a 12-pack of beer every other day as well as going to different bars. He has 100 pack-year smoking history, and currently smokes half a pack of cigarettes a day. He had also reported worsening dyspnea for three days prior to the readmission.

Admission vital signs were blood pressure, 128/95 mm Hg, pulse $98 \mathrm{bpm}$, atrial fibrillation (not new), temperature $35.2^{\circ} \mathrm{C}$, respiratory rate $20-22 / \mathrm{min}$, height $193 \mathrm{~cm}$, weight $132 \mathrm{~kg}$, pulse oximetry $98 \%$ on 2 liters per minute (LPM) nasal cannula oxygen, with a BMI of $35.4 \mathrm{~kg} / \mathrm{m}^{2}$, and pain score of 6 out of 10 for the sustained facial injuries. He had $2+$ bilateral pitting chronic edema at lower extremities. He was in respiratory distress, uncomfortable and dyspneic with increased work of breathing. Expiratory respiratory phase was prolonged, breath sounds were generally decreased with both expiratory rhonchi and bibasilar inspiratory crackles evident. He was alert and non-focal, normocephalic but showed extensive facial injuries with a large full thickness laceration originating from the right zygomatic arch and traversing medially across the nasal bridge down to the left nostril. The nose was lacerated and epistaxis was evident. There was normal jaw occlusion. Pupils were both equal, round, and reactive to light.

Admission laboratory values were as follows: Sodium 116 $\mathrm{mmol} / \mathrm{L}$ (was $134 \mathrm{mmol} / \mathrm{L}$ four days earlier), potassium $3.1 \mathrm{mmol} / \mathrm{L}$, chloride $64 \mathrm{mmol} / \mathrm{L}$, bicarbonate $35 \mathrm{mmol} / \mathrm{L}$, creatinine $0.74 \mathrm{mg} / \mathrm{dL}$, hemoglobin $14.6 \mathrm{~g} / \mathrm{dL}$, WBC 10.2 $\times 10^{9} / \mathrm{L}$, platelets $191 \times 10^{9} / \mathrm{L}$. It is noted that chloride and bicarbonate were $90 \mathrm{mmol} / \mathrm{L}$ and $37 \mathrm{mmol} / \mathrm{L}$, just four days earlier. Other laboratory values included a normal liver panel with albumin $4 \mathrm{~g} / \mathrm{dL}$ and total bilirubin 0.9 $\mathrm{mg} / \mathrm{dL}$, a recent $\mathrm{HgA} 1 \mathrm{c}$ was $6.1 \%$, and lactate was elevated at $4.7 \mathrm{mmol} / \mathrm{L}$ on admission but had normalized to 0.9 $\mathrm{mmol} / \mathrm{L}$, the next day. Troponin $\mathrm{T}$ was $0.01 \mathrm{ng} / \mathrm{mL}(<0.01$ $\mathrm{ng} / \mathrm{mL}$ ). ADH and BNP levels were not measured during this admission.

A recent echocardiogram had shown that LV ejection fraction was preserved at $60 \%$ without valvular disease and the right ventricular systolic pressure was elevated at $47 \mathrm{~mm} \mathrm{Hg}$. Chest radiograph was consistent with 
bilateral pulmonary vascular congestion. For worsening dyspnea and hypoxia, a chest CT angiogram was carried out on admission that ruled out pulmonary embolism. A non-contrast head CT examination was negative for intracranial injuries but clearly demonstrated significant right-sided facial and nasal soft tissue injuries, and a nondisplaced fracture of the right nasal bone. Emergent ENT evaluation and repair of the facial injuries were quickly completed in the emergency department (ED). For his COPD exacerbation, he was started on intravenous methylprednisone, $20 \mathrm{mg}$ every 8 hours together with albuterol/ipratropium nebulizers and biphasic positive airway pressure (BIPAP) assisted respiration with noninvasive positive airway pressure therapy as needed. Potentialalcohol withdrawal was managed using the clinical institute withdrawal assessment for alcohol, commonly abbreviated as (CIWA) protocol. Intravenous Furosemide was started initially in the emergency department and was soon discontinued for fear of worsening hyponatremia. Instead, $3 \% \mathrm{NaCl}$ infusion at $20 \mathrm{cc} / \mathrm{h}$ was initiated for symptomatic hyponatremia. Overnight, with minimal response of the hyponatremia, the dose of $3 \% \mathrm{NaCl}$ infusion was increased to $40 \mathrm{cc} / \mathrm{h}$ with the caveat that if his dyspnea from COPD exacerbation and diastolic heart failure worsened, we were going to employ the use of an $\mathrm{ADH}$ antagonist. Later the same day, dyspnea and hypoxia with respiratory distress worsened despite increased $\mathrm{FiO}_{2}$ up to 6 LPM nasal cannula oxygen. Furthermore, his bilateral lower extremity edema was worse at 3-4+, whereas hyponatremia was not improving. Moreover, a follow up chest radiograph that evening confirmed worsening pulmonary edema (Figure 1). At this stage, intravenous furosemide infusion at $20 \mathrm{mg} / \mathrm{h}$ was again considered for management of the accompanying acutely decompensating heart failure but this consideration was promptly discarded for fear of further worsening hyponatremia. Instead, he was transferred to the coronary care unit (CCU), nocturnal noninvasive positive airway pressure therapy was continued and intravenous conivaptan was started to improve hyponatremia and simultaneously achieve 'diuresis' through vaptandependent aquaresis. These outcomes would prevent further respiratory compromise and the looming need for mechanically assisted ventilation.

Serum sodium level at the time intravenous conivaptan was started was only $117 \mathrm{mmol} / \mathrm{L}$. Per the manufacturer's protocol, $20 \mathrm{mg}$ intravenous conivaptan was given as an initial loading dose over 30 minutes, followed by $20 \mathrm{mg}$ intravenous continuous infusion over 24 hours. Sodium level was monitored every four hours with the instruction to discontinue intravenous conivaptan once the sodium level was $>/=$ to $129 \mathrm{mmol} / \mathrm{L}$. The patient achieved excellent aquaresis ( $>3.5 \mathrm{~L}$ of urine) overnight with intravenous conivaptan (Figures $2 \mathrm{~A}$ and $2 \mathrm{~B}$ ). Pulmonary edema resolved promptly, both symptomatically and as confirmed in the follow up chest radiograph (Figure 1). Simultaneously, serum sodium levels responded to the conivaptan infusion and had increased to 132 $\mathrm{mmol} / \mathrm{L}$ the following morning (Figures 3 and 4 ). As a result, intravenous conivaptan infusion was promptly

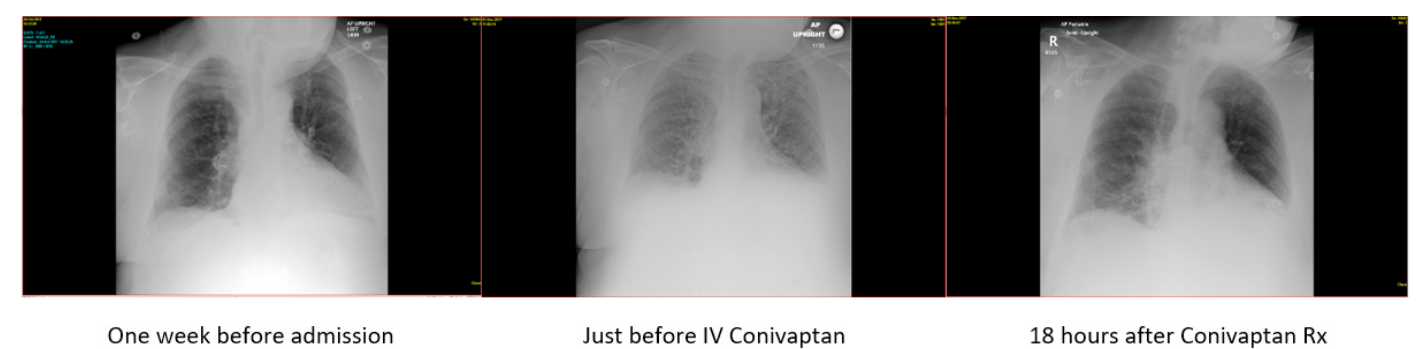

Figure 1. Composite views of chest radiograph at different timelines before and during the index admission.

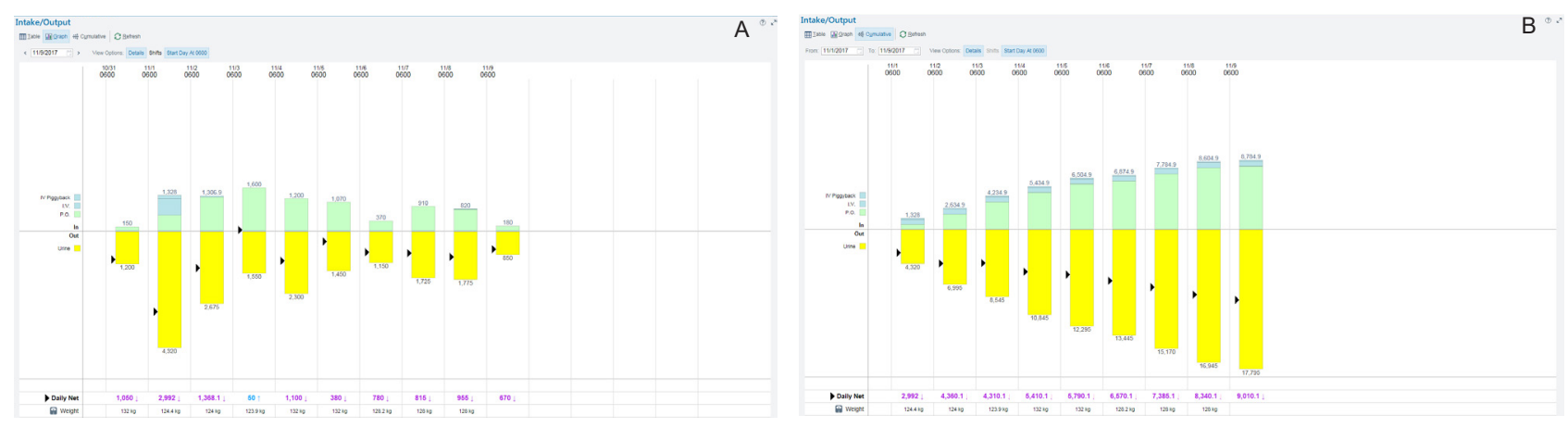

Figure 2. (A) Increased urine output (aquaresis) following intravenous Conivaptan on hospital day 2. (B) Increased cumulative urine output (aquaresis) following intravenous Conivaptan on hospital day 2. 


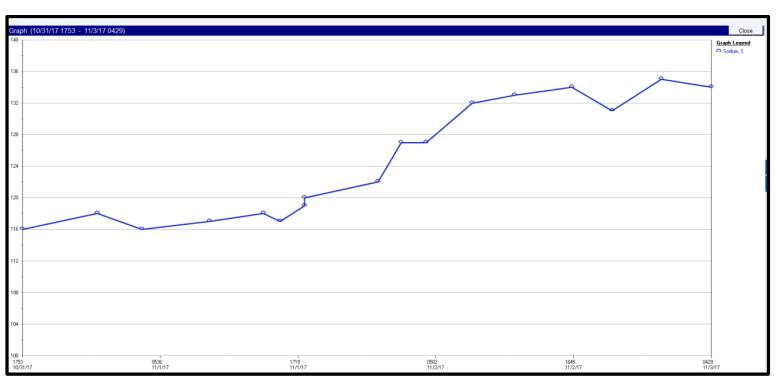

Figure 3. Response of hyponatremia to intravenous conivaptan over 12 hours.

discontinued at 830 am that morning to avoid over correction of the hyponatremia. In total intravenous conivaptan use lasted only 12 hours.

The patient was now feeling much better, dyspnea had improved remarkably and his bilateral lower extremity swelling and edema were considerably resolved. He was subsequently continued on oral furosemide, $40 \mathrm{mg}$ daily with adequate sustained urine volumes. Sodium level stabilized at about $135 \mathrm{mmol} / \mathrm{L}$ and he was discharged to a Rehab unit, otherwise asymptomatic. His admission weight was $132 \mathrm{~kg}$ and his weight on discharge was 120.5 $\mathrm{kg}$; his BMI had decreased from $35.4 \mathrm{~kg} / \mathrm{m}^{2}$ on admission to $32.3 \mathrm{~kg} / \mathrm{m}^{2}$ on discharge.

\section{Discussion}

We have presented in generally great detail, the diagnostic and therapeutic complexities involved in the management of hyponatremia especially when this is complicated by co-existing pulmonary edema, acutely decompensating heart failure and pulmonary hypertension in the context of advanced COPD. We were able to achieve excellent 'diuresis' using the aquaresis-inducing properties of intravenous conivaptan, an ADH antagonist (1-11). Notedly, dyspnea, edema and volume overload promptly resolved as confirmed by follow up symptomatic relief, resolution of physical examination findings and improvement evident in the follow up chest radiograph (Figure 1). Additionally, severe hyponatremia was corrected appropriately and promptly (Figures 3 and 4). It must be acknowledged here that overcorrection of hyponatremia, more so in situations of chronically developing hyponatremia, raises the specter of the potentially lethal syndrome of pontine demyelination (12-17). The general consensus is to correct hyponatremia at $0.5 \mathrm{mmol} / \mathrm{L} / \mathrm{h}$ and not to over correct by $>8-12 \mathrm{mmol} / \mathrm{L}$ of $\mathrm{Na}$ over 24 hours (15-17). As a result, in the management of our patient, nephrology consult instructions were very clear and firm - sodium levels were closely monitored, first after 2 hours of initiating intravenous conivaptan, then every four hours thereafter, and with the express instruction to promptly discontinue intravenous conivaptan anytime the sodium level was $129 \mathrm{mmol} / \mathrm{L}, 12 \mathrm{mmol} / \mathrm{L}$ over the sodium level when intravenous conivaptan was started.

Finally, treatment with intravenous conivaptan facilitated

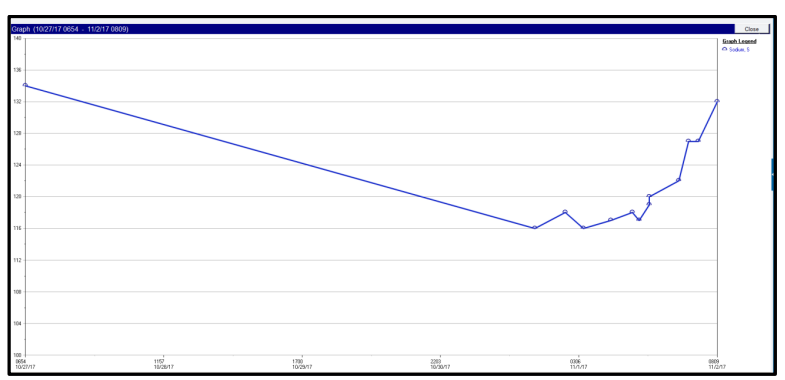

Figure 4. Serum sodium trajectory from before the index admission to the end of the intravenous conivaptan infusion.

improvement of severe symptomatic hyponatremia in our patient, and simultaneously, the resulting aquaresis led to a rapid and sustained improvement in his dyspnea from pulmonary edema. There is accruing evidence in the literature that the vaptans, intravenous conivaptan and oral tolvaptan, have now earned a place in the therapeutic armamentarium to manage symptomatic acutely decompensating heart failure complicated by severe hyponatremia and volume overload (1-11). Subsequently, the administration of loop diuretics appears to be usually well tolerated without necessarily a need for the prolonged use of the vaptans. We hope that our experience will add to the growing literature in these regards.

\section{Conclusion}

In conclusion, patients admitted with exacerbation of COPD associated with hyponatremia have a worse clinical course, and more specifically, they exhibit longer hospital stays, greater need for mechanical ventilation and an increased mortality rate (both in-hospital and in the months following discharge) (6). The increase in mortality is especially evident in those cases of severe hyponatremia. Therefore, it is prudent and mandatory to monitor hyponatremic patients, to determine the specific etiology and, although interventional studies are still needed, there is the overarching goal to normalize plasma sodium levels as soon as safely possible (6).

\section{Acknowledgments}

This work is dedicated to the memory of a very dear friend and uncle, Mr. B.C. Uchendu, who passed away back home in Lagos, Nigeria, some months ago in 2017.

\section{Authors' contribution}

MACO; Conception, design, acquisition of data, data analysis, interpretation of data, literature review, drafting the article and final approval of manuscript. RM; Acquisition of data, literature review and final approval of manuscript. NA; Literature review and final approval of manuscript. MHA; Acquisition of data, literature review and final approval of the manuscript. YS; Literature review and final approval of the manuscript. TC; Acquisition of data and final approval of the manuscript. AZ; Acquisition of data and final approval of the manuscript. AK; 
Acquisition of data, literature review and final approval of the manuscript. EA; Acquisition of data, literature review and final approval of the manuscript.

\section{Conflicts of interest}

There were no points of conflicts.

\section{Ethical considerations}

Ethical issues (including plagiarism, data fabrication, double publication) have been completely observed by the authors. The patient has given his informed consent regarding this case report.

\section{Funding/Support}

None.

\section{References}

1. Zeltser D, Rosansky S, van Rensburg H, Verbalis JG, Smith $\mathrm{N}$. Assessment of the efficacy and safety of intravenous conivaptan in euvolemic and hypervolemic hyponatremia. Am J Nephrol. 2007;27:447-57.

2. Schrier RW, Gross P, Gheorghiade M, Berl T, Verbalis JG, Czerwiec FS, et al. Tolvaptan, a selective oral vasopressin V2-receptor antagonist, for hyponatremia. N Engl J Med. 2006;355:2099-112. doi: 10.1056/NEJMoa065181

3. Rozen-Zvi B, Yahav D, Gheorghiade M, Korzets A, Leibovici L, Gafter U. Vasopressin receptor antagonists for the treatment of hyponatremia: systematic review and meta-analysis. Am J Kidney Dis 2010;56:325-37.

4. Jaber BL, Almarzouqi L, Borgi L, Seabra VF, Balk EM, Madias NE. Short term efficacy and safety of vasopressin receptor antagonists for treatment of hyponatremia. Am J Med 2010;124:977.e1-e9.

5. Ghali JK, Farah JO, Daifallah S, Zabalawi HA, Zmily HD. Conivaptan and its role in the treatment of hyponatremia. Drug Des Devel Ther. 2009;3:253-68.

6. Chalela R, González-García JG, Chillarón JJ, ValeraHernández L, Montoya-Rangel C, Badenes D, et al. Impact of hyponatremia on mortality and morbidity in patients with COPD exacerbations. Respir Med. 2016;117:237-42. doi: 10.1016/j.rmed.2016.05.003.

7. Berl T. Vasopressin antagonists. N Engl J Med. 2015;
372:2207-16. doi: 10.1056/NEJMra1403672.

8. O'Connell JB, Alemayehu A. Hyponatremia, heart failure, and the role of tolvaptan. Postgrad Med. 2012;124:29-39. doi: $10.3810 /$ pgm.2012.03.2534.

9. De Vecchis R, Cantatrione C, Mazzei D, Baldi C. Vasopressin receptor antagonists for the correction of hyponatremia in chronic heart failure: an underutilized therapeutic option in current clinical practice? J Clin Med. 2016;5(10):E86.

10. Pose A, Almenar L, Gavira JJ, López-Granados A, Blasco T, Delgado J, et al. Benefit of tolvaptan in the management of hyponatraemia in patients with diuretic-refractory congestive heart failure: the SEMI-SEC project. ESC Heart Fail. 2017;4:130-137. doi: 10.1002/ehf2.12124.

11. Vinod P, Krishnappa V, Chauvin AM, Khare A, Raina R. Cardiorenal syndrome: role of arginine vasopressin and vaptans in heart failure. Cardiol Res. 2017;8:87-95. doi: $10.14740 / \mathrm{cr} 553 \mathrm{w}$.

12. Aratani S, Hara M, Nagahama M, Taki F, Futatsuyama $\mathrm{M}$, Tsuruoka S, et al. A low initial serum sodium level is associated with an increased risk of overcorrection in patients with chronic profound hyponatremia: a retrospective cohort analysis. BMC Nephrol. 2017;18:316. doi: $\quad$ 10.1186/s12882-017-0732-1.

13. Lee A, Jo YH, Kim K, Ahn S, Oh YK, Lee H, et al. Efficacy and safety of rapid intermittent correction compared with slow continuous correction with hypertonic saline in patients with moderately severe or severe symptomatic hyponatremia: study protocol for a randomized controlled trial (SALSA trial). Trials. 2017;18:147. doi: 10.1186/ s13063-017-1865-z.

14. De Vecchis R, Noutsias M, Ariano C, Cesaro A, Cioppa C, Giasi A, et al. Does accidental overcorrection of symptomatic hyponatremia in chronic heart failure require specific therapeutic adjustments for preventing central pontine myelinolysis? J Clin Med Res. 2017; 9:266-272. doi: 10.14740/jocmr2933w.

15. Sterns RH, Hix JK, Silver SM. Management of hyponatremia in the ICU. Chest. 2013;144:672-679. doi: 10.1378/ chest.12-2600.

16. King JD, Rosner MH. Osmotic demyelination syndrome. Am J Med Sci. 2010;339:561-7.

17. Sterns RH, Riggs JE, Schochet SS Jr. Osmotic demyelination syndrome following correction of hyponatremia. N Engl J Med. 1986; 314:1535-42.

Copyright @ 2018 The Author(s); Published by Nickan Research Institute. This is an open-access article distributed under the terms of the Creative Commons Attribution License (http://creativecommons.org/licenses/by/4.0), which permits unrestricted use, distribution, and reproduction in any medium, provided the original work is properly cited. 